\title{
Rare Occurrence of the Left Maxillary Horizontal Third Molar Impaction, the Right Maxillary Third Molar Vertical Impaction and the Left Mandibular Third Molar Vertical Impaction with Inferior Alveolar Nerve Proximity in a 30 Year Old Female: A Case Report
}

\section{Karthik Shunmugavelu ${ }^{1}$}

\section{OPEN ACCESS}

Citation: Karthik Shunmugavelu. Rare Occurrence of the Left Maxillary Horizontal Third Molar Impaction, the Right Maxillary Third Molar Vertical Impaction and the Left Mandibular Third Molar Vertical Impaction with Inferior Alveolar Nerve Proximity in a 30 Year Old Female: A Case Report. Ethiop J Health Sci 2017;27(1):101-103. doi: http://dx.doi.org/10.4314/ejhs.v27i1.14.

ReceivedJune 9, 2016

Accepted: July 15, 2016

Published: January 1, 2017

Copyright: Karthik S. This is an open access article distributed under the terms of the Creative Commons Attribution License, which permits unrestricted use, distribution, and reproduction in any medium, provided the original author and source are credited.

Funding: Nil

Competing Interests: The authors declare that this manuscript was approved by all authors in its form and that no competing interest exists.

Affiliation and Correspondence:

${ }^{1}$ Mercy Multispeciality Dental Centre,

Tamilnadu, India

*Email: drkarthiks1981@gmail.com

\begin{abstract}
BACKGROUND: A combination of horizontal and vertical third molar impaction is a rare occurrence. When the tooth is unable to erupt to its proper position and fail to achieve a normal occlusion, it is known as impacted tooth.

CASE DETAILS: In this scientific article, case report of a female patient aged 30 years reported with acombination of horizontally and vertically impacted third molars in the maxilla and mandible has been presented. The treatment included surgical removal of the impacted teeth without any damage to underlying structures.

CONCLUSION: Horizontal impaction of left maxillary third molar,vertical impaction of right maxillary third molar and left mandibular third molar is a rare occurrence. If symptomatic, surgical removal has to be planned as earlier as possible rather than late complications.
\end{abstract}

\section{INTRODUCTION}

Impaction of tooth is a commonly seen $(2,4)$. When the tooth is unable to erupt to its proper position and fails to achieve a normal occlusion, it is known as impacted tooth. Etiology includescrowding, ectopic position of the tooth germ, supernumerary teeth and soft tissue or bony lesions. Most commonly, impacted teeth accounts to maxillary and mandibular third molars $(3,5)$. Pathogenesis relates to the discrepancy of jaw size to the tooth (1). A rare case of horizontal impaction of the left maxillary third molar,vertical impaction of right maxillary third molar and left mandibular third molar in a 30 year old female has been reported.

\section{CASE DETAILS}

A 30-year old female presented to the Department of Dentistry and Faciomaxillary Surgery complaining of discomfort in the right maxillary third molar region, left maxillary third molar region and left mandibular third molar region. General health 
examination revealed that the patient was conscious, oriented and afebrile, and the vitals were stable. On intraoral clinical examination, all the three mentioned regions revealed absence of clincial crown exposure. The patient stated that the discomfort had been present since a week. Previous dental history revealed that the left mandibular second molar and the right mandibular third molar were extracted in a conventional manner and surgical method respectively before two years. The pain increased in intensity during mastication. Radiographically, horizontal impaction of the left maxillary third molar, vertical impaction of the right maxillary third molar and the left mandibular third molar was seen in Orthopantomogram (OPG) (Figure 1). The treatment procedure included planned surgical removal of the specific tooth under general anesthesia (GA). Under GA, the patient was draped, preparation was done, the mouth was propped and Fraser suction was in place, topical local anesthetic was applied, incision was placed with the no.3 and no.11 Bard Parker surgical handle and blade, mucoperiosteal flap was raised with the help of periosteal elevator, overlying bone and the vertically impacted right maxillary third molar tooth clinically were seen. The flap was retracted with the help of Austins retractor whereas the tongue-by-tongue depressor.With the help of micromotor handpiece apparatus which includes high speed sharp carbide burs, no.557/703 fissure burs, no. 8 round bur, excess bone coverage was trimmed so that the vertically placed tooth was luxated in a slow manner and the whole tooth removed by maxillary third molar forceps. Saline irrigation was done with 18 gauge needle and $10 \mathrm{ml}$ syringe to reduce the heat production during bone cutting. The socket was curetted for fresh bleeding, bony margins were trimmed by bone rongeur, bone smoothening ws done by bone file, mucoperiosteal flap was replaced, soft tissue was held by Adson forceps and 3-0 black silk sutures were placed with the help of needle holder along with cutting edge suture needle and excess suture material was removed by Metzenbaum scissors. After a week of review, the sutures were removed with an evidence of excellent intraoral healing. A similiar procedure was done in relation to the left maxillary horizontal third molar impaction and the left mandibular vertical third molar impaction.

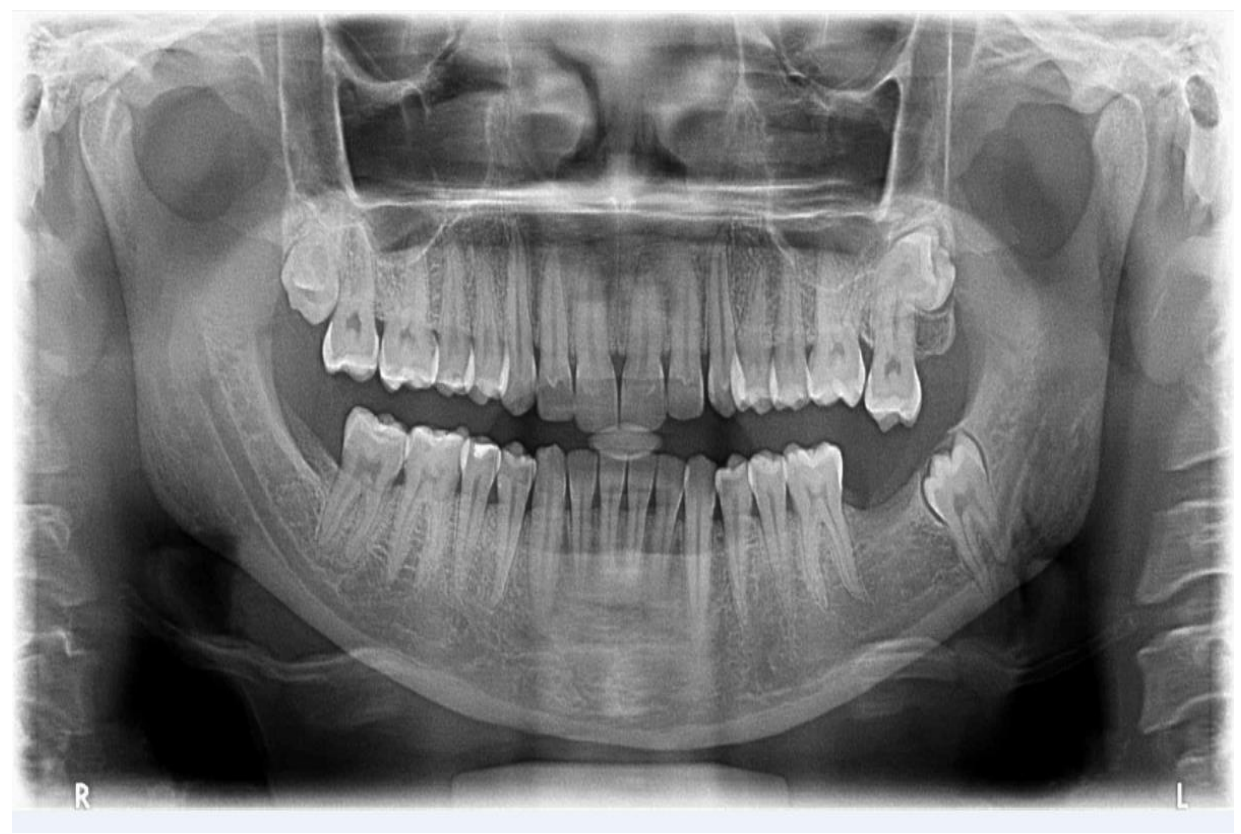

Figure 1: Orthopantomogram depicting maxillary right vertically impacted third molar(arrowhead), maxillary left horizontally impacted third molar(arrowhead), mandibular left vertically impacted third molar(arrowhead)

DOI: http://dx.doi.org/10.4314/ejhs.v27i1.14 


\section{DISCUSSION}

Impaction of tooth is commonly seen involving mandibular third molars followed by maxillary third molars (5). If left undiagnosed and untreated, sequential events such as periodontal disease, dental caries, odontogenic cyst and tumors, pain of unexplained origin, jaw fracture and resorption of root of the adjacent tooth might occur (6) occur. Types of impaction include vertical, horizontal, mesioangular, distoangular and even inverted impaction. In complete submerged horizontal or vertical tooth impactions, a greater amount of bone guttering needs to be done, thereby leading to an increase in the size of the socket. Careful consideration has to be given in order to avoid incidents such as tooth displacement into infratemporal space and pterygomandibular space, excess bleeding, and displacement of tooth into maxillary sinus and alveolar fracture. Horizontal impaction of the left maxillary third molar,vertical impaction of the right maxillary third molar and the left mandibular third molar is a rare occurrence. If symptomatic, surgical removal has to be planned as earlier as possible rather to avod late complications.

\section{REFERENCES}

1. Togoo R A Rare occurrence of Inverted Maxillary Third Molar.Case Report. Journal of International Oral Health. May-June 2013; 5(3):85-87.

2. Gisakis IG, Palamidakis FD, Farmakis ER, Kamberos G, Kamberos S. Prevalence of impacted teeth in a Greek population. Journal of Investigative and Clinical Dentistry 2011;2:1-8.

3. Mwaniki D, Guthua SW. Incidence of impacted mandibular third molars among dental patients in Nairobi, Kenya. Odontostomatol Trop 17-9.

4. Chu FCS, Li TKL, Lui VKB, Newsome PRH, Chow RLK, Cheung LK. Prevalence of impacted teeth and associated pathologies - a radiographic study of the Hong Kong Chinese population. Hong Kong Med J. 2003;9(3):158.

5. Al-Faleh W. Completely Impacted teeth in dentate and edentulous Jaws, Pakistan Oral and Dental Journal 2009;29: 255-60.

6. Maglutac M, Sarmiento MA, Echiverre N. Impacted Maxillary Premolar: A Report of Two Cases. Emilio Aguinaldo College Research Bulletin 2008;7(1):15-21. 